\title{
BOUNDARY VALUE PROBLEMS FOR ELLIPTIC INTEGRO-DIFFERENTIAL OPERATORS
}

\author{
KAZUAKI TAIRA \\ Institute of Mathematics, University of Tsukuba, Tsukuba 305, JAPAN \\ Dedicated to Professor Kiyosi Itô on his 80th birthday
}

\section{Introduction and results.}

This paper is a continuation of the previous note [T2] where we studied a class of degenerate boundary value problems for second-order elliptic differential operators and proved that this class of boundary value problems generates analytic semigroups both in the $L^{p}$ topology and in the topology of uniform convergence. The purpose of this paper is to extend these results to the elliptic integro-differential operator case.

Let $D$ be a bounded, convex domain of Euclidean space $\mathbf{R}^{N}$, with $C^{\infty}$ boundary $\partial D$; its closure $\bar{D}=D \cup \partial D$ is an $N$-dimensional, compact $C^{\infty}$ manifold with boundary.

Let $W$ be a second-order, elliptic integro-differential operator with real coefficients such that

$$
\begin{aligned}
W u(x) & =A u(x)+S u(x) \\
& \left.:=\int_{i, j=1}^{N} a^{i j}(x) \frac{\partial^{2} u}{\partial x_{i} \partial x_{j}}(x)+\sum_{i=1}^{N} b^{i}(x) \frac{\partial u}{\partial x_{i}}(x)+c(x) u(x)\right) \\
& +\int_{\mathbf{R}^{N} \backslash\{0\}}\left(u(x+z)-u(x)-\sum_{j=1}^{N} z_{j} \frac{\partial u}{\partial x_{j}}(x)\right) s(x, z) m(d z) .
\end{aligned}
$$

Here:

(1) $a^{i j} \in C^{\infty}(\bar{D}), a^{i j}=a^{j i}$ and there exists a constant $a_{0}>0$ such that

$$
\sum_{i, j=1}^{N} a^{i j}(x) \xi_{i} \xi_{j} \geq a_{0}|\xi|^{2}, \quad x \in D, \xi \in \mathbf{R}^{N}
$$

(2) $b^{i} \in C^{\infty}(\bar{D})$.

(3) $c \in C^{\infty}(\bar{D})$, and $c \leq 0$ in $D$ but $c \not \equiv 0$ in $D$.

(4) $s \in C\left(\bar{D} \times \mathbf{R}^{N}\right)$ and $0 \leq s \leq 1$ in $D \times \mathbf{R}^{N}$, and there exist constants $C_{0}>0$ and $0<\theta_{0}<1$ such that

$$
|s(x, z)-s(y, z)| \leq C_{0}|x-y|^{\theta_{0}}, \quad x, y \in D, z \in \mathbf{R}^{N}
$$


and

$$
s(x, z)=0 \quad \text { if } x+z \notin \bar{D} .
$$

Condition (0.1) implies that the integral operator $S$ may be considered as an operator acting on functions $u$ defined on the closure $\bar{D}$ (see [G-M, Chapter II, Remark $1.19])$.

(5) The measure $m(d z)$ is a Radon measure on $\mathbf{R}^{N} \backslash\{0\}$ such that

$$
\int_{\{|z| \leq 1\}}|z|^{2} m(d z)+\int_{\{|z|>1\}}|z| m(d z)<\infty .
$$

The operator $W$ is called a second-order Waldenfels operator. The differential operator $A$ is called a diffusion operator which describes analytically a strong Markov process with continuous paths in the interior $D$. The integral operator $S$ is called a second-order Lévy operator which is supposed to correspond to the jump phenomenon in the closure $\bar{D}$ (cf. [B-C-P], [T1]).

Let $L$ be a first-order, boundary condition with real coefficients such that

$$
L u\left(x^{\prime}\right)=\mu\left(x^{\prime}\right) \frac{\partial u}{\partial \mathbf{n}}\left(x^{\prime}\right)+\gamma\left(x^{\prime}\right) u\left(x^{\prime}\right) .
$$

Here:

(1) $\mu \in C^{\infty}(\partial D)$ and $\mu \geq 0$ on $\partial D$.

(2) $\gamma \in C^{\infty}(\partial D)$ and $\gamma \leq 0$ on $\partial D$.

(3) $\mathbf{n}=\left(n_{1}, n_{2}, \ldots, n_{N}\right)$ is the unit interior normal to the boundary $\partial D$.

The boundary condition $L$ is called a first-order Ventcel' boundary condition. The terms $\mu \partial u / \partial \mathbf{n}$ and $\gamma u$ of $L$ are supposed to correspond to the reflection phenomenon and the absorption phenomenon, respectively.

Our fundamental hypothesis is the following:

(H) $\mu\left(x^{\prime}\right)-\gamma\left(x^{\prime}\right)>0$ on $\partial D$.

The intuitive meaning of hypothesis $(\mathrm{H})$ is that either the reflection phenomenon or the absorption phenomenon occurs at each point of the boundary $\partial D$.

The first purpose of this paper is to prove an existence and uniqueness theorem for the following nonhomogeneous boundary value problem in the framework of Hölder spaces:

$$
\begin{cases}W u=f & \text { in } D, \\ L u=\varphi & \text { on } \partial D .\end{cases}
$$

The crucial point is how to define a version of Hölder spaces in which problem $(*)$ is uniquely solvable.

We introduce a subspace of the Hölder space $C^{1+\theta}(\partial D), 0<\theta<1$, which is associated with the boundary condition $L$ in the following way: We let

$$
C_{L}^{1+\theta}(\partial D)=\left\{\varphi=\mu \varphi_{1}-\gamma \varphi_{2}: \varphi_{1} \in C^{1+\theta}(\partial D), \varphi_{2} \in C^{2+\theta}(\partial D)\right\},
$$

and define a norm

$$
|\varphi|_{C_{L}^{1+\theta}(\partial D)}=\inf \left\{\left|\varphi_{1}\right|_{C^{1+\theta}(\partial D)}+\left|\varphi_{2}\right|_{C^{2+\theta}(\partial D)}: \varphi=\mu \varphi_{1}-\gamma \varphi_{2}\right\} .
$$


Then it is easy to verify that the space $C_{L}^{1+\theta}(\partial D)$ is a Banach space with respect to the norm $|\cdot|_{C_{L}^{1+\theta}(\partial D)}$. We remark that the space $C_{L}^{1+\theta}(\partial D)$ is an "interpolation space" between $C^{2+\theta}(\partial D)$ and $C^{1+\theta}(\partial D)$. More precisely, we have

$$
\begin{cases}C_{L}^{1+\theta}(\partial D)=C^{2+\theta}(\partial D) & \text { if } \mu \equiv 0 \text { on } \partial D \\ C_{L}^{1+\theta}(\partial D)=C^{1+\theta}(\partial D) & \text { if } \mu>0 \text { on } \partial D .\end{cases}
$$

Now we can state our existence and uniqueness theorem for problem $(*)$ :

Theorem 1. If hypothesis $(H)$ is satisfied, then the mapping

$$
(W, L): C^{2+\theta}(\bar{D}) \longrightarrow C^{\theta}(\bar{D}) \oplus C_{L}^{1+\theta}(\partial D)
$$

is an algebraic and topological isomorphism for all $0<\theta<\theta_{0}$. In particular, for any $f \in C^{\theta}(\bar{D})$ and any $\varphi \in C_{L}^{1+\theta}(\partial D)$, there exists a unique solution $u \in C^{2+\theta}(\bar{D})$ of problem $(*)$.

As an application of Theorem 1, we consider the problem of existence of Markov processes in probability theory. To do so, we let

$$
M=\left\{x^{\prime} \in \partial D: \mu\left(x^{\prime}\right)=0\right\} .
$$

Then, in view of condition $(\mathrm{H})$ it follows that the boundary condition $L u=0$ on $\partial D$ includes the condition $u=0$ on $M$. With this fact in mind, we let

$$
C_{0}(\bar{D} \backslash M)=\{u \in C(\bar{D}): u=0 \text { on } M\} .
$$

The space $C_{0}(\bar{D} \backslash M)$ is a closed subspace of $C(\bar{D})$; hence it is a Banach space.

A strongly continuous semigroup $\left\{T_{t}\right\}_{t \geq 0}$ on the space $C_{0}(\bar{D} \backslash M)$ is called a Feller semigroup on $\bar{D} \backslash M$ if it is non-negative and contractive on $C_{0}(\bar{D} \backslash M)$ :

$$
f \in C_{0}(\bar{D} \backslash M), 0 \leq f \leq 1 \quad \text { on } \bar{D} \backslash M \Longrightarrow 0 \leq T_{t} f \leq 1 \quad \text { on } \bar{D} \backslash M .
$$

It is known (cf. [T1, Chapter 9]) that if $T_{t}$ is a Feller semigroup on $\bar{D} \backslash M$, then there exists a unique Markov transition function $p_{t}$ on $\bar{D} \backslash M$ such that

$$
T_{t} f(x)=\int_{\bar{D} \backslash M} p_{t}(x, d y) f(y), \quad f \in C_{0}(\bar{D} \backslash M),
$$

and further $p_{t}$ is the transition function of some strong Markov process.

We define a linear operator $\mathcal{W}$ from $C_{0}(\bar{D} \backslash M)$ into itself as follows:

(a) The domain of definition $D(\mathcal{W})$ is the set

$$
D(\mathcal{W})=\left\{u \in C^{2}(\bar{D}) \cap C_{0}(\bar{D} \backslash M): W u \in C_{0}(\bar{D} \backslash M), L u=0\right\} .
$$

(b) $\mathcal{W} u=W u, u \in D(\mathcal{W})$.

The next theorem is a generalization of Theorem 4 of [T2] to the integrodifferential operator case: 
Theorem 2. If hypothesis $(H)$ is satisfied, then the operator $\mathcal{W}$ is closable in the space $C_{0}(\bar{D} \backslash M)$, and its minimal closed extension $\overline{\mathcal{W}}$ is the infinitesimal generator of some Feller semigroup $\left\{T_{t}\right\}_{t \geq 0}$ on $\bar{D} \backslash M$.

Theorem 2 asserts that there exists a Feller semigroup on $\bar{D} \backslash M$ corresponding to such a diffusion phenomenon that a Markovian particle moves both by jumps and continuously in the state space $\bar{D} \backslash M$ until it "dies" at the time when it reaches the set $M$ where the particle is definitely absorbed (cf. [K, Theorem 5.2], [S, Theorem 2.2], [G-M, Chapter VIII, Theorem 3.3]).

The second purpose of this paper is to study problem $(*)$ from the point of view of analytic semigroup theory in functional analysis. The forthcoming two theorems generalize Theorems 2 and 3 of [T2] to the integro-differential operator case.

First we state a generation theorem of analytic semigroups in the the $L^{p}$ topology. To do so, we associate with problem $(*)$ a unbounded linear operator $W_{p}$ from $L^{p}(D)$ into itself as follows:

(a) The domain of definition $D\left(W_{p}\right)$ is the set

$$
D\left(W_{p}\right)=\left\{u \in H^{2, p}(D): L u=0\right\} .
$$

(b) $W_{p} u=W u, u \in D\left(W_{p}\right)$.

Then we can prove the following:

Theorem 3. Let $1<p<\infty$. Assume that hypothesis $(H)$ is satisfied. Then we have the following:

(i) For every $\varepsilon>0$, there exists a constant $r_{p}(\varepsilon)>0$ such that the resolvent set of $W_{p}$ contains the set $\Sigma_{p}(\varepsilon)=\left\{\lambda=r^{2} e^{i \vartheta}: r \geq r_{p}(\varepsilon),-\pi+\varepsilon \leq \vartheta \leq \pi-\varepsilon\right\}$, and that the resolvent $\left(W_{p}-\lambda I\right)^{-1}$ satisfies the estimate

$$
\left\|\left(W_{p}-\lambda I\right)^{-1}\right\| \leq \frac{c_{p}(\varepsilon)}{|\lambda|}, \quad \lambda \in \Sigma_{p}(\varepsilon),
$$

where $c_{p}(\varepsilon)>0$ is a constant depending on $\varepsilon$.

(ii) The operator $W_{p}$ generates a semigroup $e^{z W_{p}}$ on the space $L^{p}(D)$ which is analytic in the sector $\Delta_{\varepsilon}=\{z=t+i s: z \neq 0,|\arg z|<\pi / 2-\varepsilon\}$ for any $0<\varepsilon<$ $\pi / 2$.

Secondly we state a generation theorem of analytic semigroups in the topology of uniform convergence. We introduce a linear operator $\mathfrak{W}$ from $C_{0}(\bar{D} \backslash M)$ into itself as follows:

(a) The domain of definition $D(\mathfrak{W})$ is the set

$$
D(\mathfrak{W})=\left\{u \in C_{0}(\bar{D} \backslash M) \cap H^{2, p}(D): W u \in C_{0}(\bar{D} \backslash M), L u=0\right\} .
$$

(b) $\mathfrak{W} u=W u, u \in D(\mathfrak{W})$.

Here we remark that the domain $D(\mathfrak{W})$ is independent of $N<p<\infty$ (see the proof of Lemma 4.2).

Then Theorem 3 remains valid with $L^{p}(D)$ and $W_{p}$ replaced by $C_{0}(\bar{D} \backslash M)$ and $\mathfrak{W}$, respectively: 
Theorem 4. If hypothesis $(H)$ is satisfied, then we have the following:

(i) For every $\varepsilon>0$, there exists a constant $r(\varepsilon)>0$ such that the resolvent set of $\mathfrak{W}$ contains the set $\Sigma(\varepsilon)=\left\{\lambda=r^{2} e^{i \vartheta}: r \geq r(\varepsilon),-\pi+\varepsilon \leq \vartheta \leq \pi-\varepsilon\right\}$, and that the resolvent $(\mathfrak{W}-\lambda I)^{-1}$ satisfies the estimate

$$
\left\|(\mathfrak{W}-\lambda I)^{-1}\right\| \leq \frac{c(\varepsilon)}{|\lambda|}, \quad \lambda \in \Sigma(\varepsilon),
$$

where $c(\varepsilon)>0$ is a constant depending on $\varepsilon$.

(ii) The operator $\mathfrak{W}$ generates a semigroup $e^{z \mathfrak{W}}$ on the space $C_{0}(\bar{D} \backslash M)$ which is analytic in the sector $\Delta_{\varepsilon}=\{z=t+i s: z \neq 0,|\arg z|<\pi / 2-\varepsilon\}$ for any $0<$ $\varepsilon<\pi / 2$.

Theorems 3 and 4 express a regularizing effect for the parabolic integro-differential operator $\partial / \partial t-W$ with homogeneous boundary condition $L$ (cf. [G-M, Chapter VIII, Theorem 3.1]).

The rest of this paper is organized as follows. In Section 1 we study problem (*) in the framework of Hölder spaces, and prove Theorem 1. The essential point in the proof is to estimate the integral operator $S$ in terms of Hölder norms. We show that the operator $(W, L)$ may be considered as a perturbation of a compact operator to the operator $(A, L)$ in the framework of Hölder spaces. Thus the proof of Theorem 1 is reduced to the differential operator case which is studied in detail in [T2]. Section 2 is devoted to the proof of Theorem 2. The proof is based on a version of the Hille-Yosida theorem in semigroup theory in terms of the maximum principle. In Section 3 we prove Theorem 3. We estimate the integral operator $S$ in terms of $L^{p}$ norms, and show that $S$ is an $A_{p}$-completely continuous operator in the sense of Gohberg and Krel̆ [G-K]. Section 4 is devoted to the proof of Theorem 4. Theorem 4 follows from Theorem 3 by using Sobolev's imbedding theorems and a $\lambda$-dependent localization argument, just as in [T2].

\section{Proof of Theorem 1.}

(I) First we prove Theorem 1 in the case where $S \equiv 0$ :

Theorem 1.1. If hypothesis $(H)$ is satisfied, then the mapping

$$
(A, L): C^{2+\theta}(\bar{D}) \longrightarrow C^{\theta}(\bar{D}) \oplus C_{L}^{1+\theta}(\partial D)
$$

is an algebraic and topological isomorphism for all $0<\theta<1$.

Proof. The proof is divided into four steps.

(i) Let $(f, \varphi)$ be an arbitrary element of $C^{\theta}(\bar{D}) \oplus C_{L}^{1+\theta}(\partial D)$ with $\varphi=\mu \varphi_{1}-\gamma \varphi_{2}$. First we show that the boundary value problem

$$
\begin{cases}A u=f & \text { in } D, \\ L u=\varphi & \text { on } \partial D\end{cases}
$$

can be reduced to the study of an operator on the boundary.

To do so, we consider the following Neumann problem:

$$
\begin{cases}A v=f & \text { in } D \\ \frac{\partial v}{\partial \mathbf{n}}=\varphi_{1} & \text { on } \partial D .\end{cases}
$$


Recall that the existence and uniqueness theorem for problem $(N)$ is well established in the framework of Hölder spaces (see [G-T, Theorem 6.31]). Thus we find that a function $u \in C^{2+\theta}(\bar{D})$ is a solution of problem $(*)$ if and only if the function $w=u-v \in C^{2+\theta}(\bar{D})$ is a solution of the problem:

$$
\begin{cases}A w=0 & \text { in } D, \\ L w=\varphi-L v & \text { on } \partial D .\end{cases}
$$

Here we remark that

$$
L v=\mu \frac{\partial v}{\partial \mathbf{n}}+\gamma v=\mu \varphi_{1}+\gamma v
$$

so that

$$
L w=-\gamma\left(\varphi_{2}+v\right) \in C^{2+\theta}(\partial D) .
$$

However we know that every solution $w \in C^{2+\theta}(\bar{D})$ of the homogeneous equation: $A w=0$ in $D$ can be expressed as follows (see [G-T, Theorem 6.14]):

$$
w=\mathcal{P} \psi, \quad \psi \in C^{2+\theta}(\partial D) .
$$

Thus one can reduce the study of problem $(* *)$ to that of the equation

$$
T \psi:=L \mathcal{P} \psi=-\gamma\left(\varphi_{2}+v\right) \quad \text { on } \partial D
$$

More precisely we have the following:

Proposition 1.2. For functions $f \in C^{\theta}(\bar{D})$ and $\varphi \in C_{L}^{1+\theta}(\partial D)$, there exists a solution $u \in C^{2+\theta}(\bar{D})$ of problem $(* *)$ if and only if there exists a solution $\psi \in$ $C^{2+\theta}(\partial D)$ of equation $(+)$.

(ii) We study the operator $T$ in question. It is known (cf. $[\mathrm{H}$, Chapter XX]) that the operator

$$
T \psi=L \mathcal{P} \psi=\mu \frac{\partial}{\partial \mathbf{n}}(\mathcal{P} \psi)+\gamma \psi
$$

is a first-order, pseudo-differential operator on the boundary $\partial D$.

The next proposition is an essential step in the proof of Theorem 1.1:

Proposition 1.3. If hypothesis $(H)$ is satisfied, then there exists a parametrix $E$ in the Hörmander class $L_{1,1 / 2}^{0}(\partial D)$ for $T$ which maps $C^{k+\theta}(\partial D)$ continuously into itself for any integer $k \geq 0$.

Proof. By making use of Theorem 22.1.3 of [H, Chapter XXII] just as in [T2, Lemma 4.2], one can construct a parametrix $E$ in the Hörmander class $L_{1,1 / 2}^{0}(\partial D)$ for $T$ :

$$
E T \equiv T E \equiv I \quad \bmod L^{-\infty}(\partial D)
$$

The boundedness of $E: C^{k+\theta}(\partial D) \rightarrow C^{k+\theta}(\partial D)$ follows from an application of [B, Theorem 1], since $C^{k+\theta}(\partial D)=B_{\infty, \infty}^{k+\theta}(\partial D)$.

(iii) We consider problem $(* *)$ in the framework of Sobolev spaces of $L^{p}$ style, and prove an $L^{p}$ version of Theorem 1.1. 
If $k$ is a positive integer and $1<p<\infty$, we define the Sobolev space

$$
\begin{aligned}
H^{k, p}(D)= & \text { the space of (equivalence classes of) functions } \\
& u \in L^{p}(D) \text { whose derivatives } D^{\alpha} u,|\alpha| \leq k, \text { in the } \\
& \text { sense of distributions are in } L^{p}(D),
\end{aligned}
$$

and the Besov space

$$
\begin{aligned}
B^{k-1 / p, p}(\partial D)= & \text { the space of the boundary values } \varphi \text { of functions } \\
& u \in H^{k, p}(D)
\end{aligned}
$$

In the space $B^{k-1 / p, p}(\partial D)$, we introduce a norm

$$
|\varphi|_{B^{k-1 / p, p}(\partial D)}=\inf \|u\|_{H^{k, p}(D)}
$$

where the infimum is taken over all functions $u \in H^{k, p}(D)$ which equal $\varphi$ on the boundary $\partial D$. The space $B^{k-1 / p, p}(\partial D)$ is a Banach space with respect to this norm $|\cdot|_{B^{k-1 / p, p}(\partial D)}($ cf. [B-L]).

We introduce a subspace of $B^{1-1 / p, p}(\partial D)$ which is an $L^{p}$ version of $C_{L}^{1+\theta}(\partial D)$. We let

$$
\begin{aligned}
B_{L}^{1-1 / p, p}(\partial D)= & \left\{\varphi=\mu \varphi_{1}-\gamma \varphi_{2}:\right. \\
& \left.\varphi_{1} \in B^{1-1 / p, p}(\partial D), \varphi_{2} \in B^{2-1 / p, p}(\partial D)\right\}
\end{aligned}
$$

and define a norm

$$
|\varphi|_{B_{L}^{1-1 / p, p}(\partial D)}=\inf \left\{\left|\varphi_{1}\right|_{B^{1-1 / p, p}(\partial D)}+\left|\varphi_{2}\right|_{B^{2-1 / p, p}(\partial D)}: \varphi=\mu \varphi_{1}-\gamma \varphi_{2}\right\} .
$$

Then it is easy to verify that the space $B_{L}^{1-1 / p, p}(\partial D)$ is a Banach space with respect to the norm $|\cdot|_{B_{L}^{1-1 / p, p}(\partial D)}$.

Then, arguing just as in the proof of [T2, Theorem 1] we can obtain the following $L^{p}$ version of Theorem 1.1:

Theorem 1.4. If hypothesis (H) is satisfied, then the mapping

$$
(A, L): H^{2, p}(D) \longrightarrow L^{p}(D) \oplus B_{L}^{1-1 / p, p}(\partial D)
$$

is an algebraic and topological isomorphism.

(iv) Now we remark that

$$
\left\{\begin{array}{l}
C^{\theta}(\bar{D}) \subset L^{p}(D) \\
C_{L}^{1+\theta}(\partial D) \subset B_{L}^{1-1 / p, p}(\partial D)
\end{array}\right.
$$


Thus we find from Theorem 1.4 that problem $(* *)$ has a unique solution $u \in$ $H^{2, p}(D)$ for any $f \in C^{\theta}(\bar{D})$ and any $\varphi \in C_{L}^{1+\theta}(\partial D)$. Furthermore, by virtue of Proposition 1.2 it follows that the solution $u$ can be written in the form

$$
u=v+\mathcal{P} \psi, \quad v \in C^{2+\theta}(\bar{D}), \psi \in B^{2-1 / p, p}(\partial D) .
$$

However, Proposition 1.3 tells us that

$$
\psi \in C^{2+\theta}(\partial D)
$$

since we have $\psi \equiv E(T \psi)=-E\left(\gamma\left(\varphi_{2}+v\right)\right) \bmod C^{\infty}(\partial D)$.

Therefore we obtain that

$$
u=v+\mathcal{P} \psi \in C^{2+\theta}(\bar{D}) .
$$

The proof of Theorem 1.1 is complete.

(II) Next we study the integral operator $S$ in the framework of Hölder spaces. To do so, we need the following elementary estimates for the measure $m(d z)$ :

Claim 1.5. For $\varepsilon>0$, we let

$$
\begin{aligned}
\sigma(\varepsilon) & =\int_{\{|z| \leq \varepsilon\}}|z|^{2} m(d z), \\
\delta(\varepsilon) & =\int_{\{|z|>\varepsilon\}}|z| m(d z) \\
\tau(\varepsilon) & =\int_{\{|z|>\varepsilon\}} m(d z)
\end{aligned}
$$

Then we have, as $\varepsilon \downarrow 0$,

$$
\begin{aligned}
& \sigma(\varepsilon) \rightarrow 0, \\
& \delta(\varepsilon) \leq \frac{C_{1}}{\varepsilon}+C_{2}, \\
& \tau(\varepsilon) \leq \frac{C_{1}}{\varepsilon^{2}}+C_{2},
\end{aligned}
$$

where

$$
C_{1}=\int_{\{|z| \leq 1\}}|z|^{2} m(d z), \quad C_{2}=\int_{\{|z|>1\}}|z| m(d z) .
$$

Proof. Assertion (1.1) follows immediately from condition (0.2).

The term $\delta(\varepsilon)$ can be estimated as follows:

$$
\begin{aligned}
\delta(\varepsilon) & =\int_{\{|z|>1\}}|z| m(d z)+\int_{\{\varepsilon<|z| \leq 1\}}|z| m(d z) \\
& \leq \int_{\{|z|>1\}}|z| m(d z)+\frac{1}{\varepsilon} \int_{\{\varepsilon<|z| \leq 1\}}|z|^{2} m(d z) \\
& \leq \int_{\{|z|>1\}}|z| m(d z)+\frac{1}{\varepsilon} \int_{\{|z| \leq 1\}}|z|^{2} m(d z) .
\end{aligned}
$$

The term $\tau(\varepsilon)$ is estimated in a similar way.

By virtue of Claim 1.5, we can estimate the term $S u$ in terms of Hölder norms, just as in [G-M, Chapter II, Lemmas 1.2 and 1.5]: 
Lemma 1.6. For every $\eta>0$, there exists a constant $C_{\eta}>0$ such that we have, for all $u \in C^{2}(\bar{D})$,

$$
\|S u\|_{\infty} \leq \eta\left\|\nabla^{2} u\right\|_{\infty}+C_{\eta}\left(\|u\|_{\infty}+\|\nabla u\|_{\infty}\right)
$$

Here

$$
\|u\|_{\infty}=\sup _{x \in D}|u(x)|
$$

Lemma 1.7. For every $\eta>0$, there exists a constant $C_{\eta}>0$ such that we have, for all $u \in C^{2+\theta_{0}}(\bar{D})$,

$$
\|S u\|_{C^{\theta_{0}}(\bar{D})} \leq \eta\left\|\nabla^{2} u\right\|_{C^{\theta_{0}}(\bar{D})}+C_{\eta}\left(\|u\|_{C^{\theta_{0}}(\bar{D})}+\|\nabla u\|_{C^{\theta_{0}}(\bar{D})}\right) .
$$

Here

$$
\|u\|_{C^{\theta_{0}}(\bar{D})}=\|u\|_{\infty}+[u]_{\theta_{0}}, \quad[u]_{\theta_{0}}=\sup _{\substack{x, y \in D \\ x \neq y}} \frac{|u(x)-u(y)|}{|x-y|^{\theta_{0}}} .
$$

(III) End of Proof of Theorem 1. First, Theorem 1.1 implies that

$$
\text { ind }(A, L)=0 \text {. }
$$

On the other hand, Lemma 1.7 tells us that the operator $S$ maps $C^{2+\theta_{0}}(\bar{D})$ continuously into $C^{\theta_{0}}(\bar{D})$. Hence it follows from an application of [B-C-P, Théorème $\mathrm{XXII}]$ that $S$ is a compact operator from $C^{2+\theta}(\bar{D})$ into $C^{\theta}(\bar{D})$ for all $0<\theta<\theta_{0}$. This implies that the operator $(W, L)$ is a perturbation of a compact operator to the operator $(A, L)$.

Hence we find that

$$
\text { ind }(W, L)=\operatorname{ind}(A, L)=0 \text {. }
$$

Therefore, in order to show the bijectivity of $(W, L)$ it suffices to prove its injectivity:

$$
\left\{\begin{array}{l}
u \in C^{2+\theta}(\bar{D}), W u=0 \text { in } D, L u=0 \text { on } \partial D \\
\Longrightarrow u=0 \text { in } D .
\end{array}\right.
$$

However, this is an immediate consequence of the following maximum principle:

Proposition 1.8. If hypothesis $(H)$ is satisfied, then we have:

$$
\left\{\begin{array}{l}
u \in C^{2}(\bar{D}), W u \geq 0 \text { in } D, L u \geq 0 \text { on } \partial D \\
\Longrightarrow u \leq 0 \text { on } \bar{D} .
\end{array}\right.
$$

Proof. If $u$ is a constant $m$, then we have $0 \leq W u=m c$ in $D$. This implies that $u \equiv m$ is non-positive, since $c \leq 0$ and $c \not \equiv 0$ in $D$.

Now we consider the case where $u$ is not a constant. Assume to the contrary that:

$$
m=\max _{\bar{D}} u>0
$$


Then, applying the strong maximum principle (see [B-C-P, Théorème VII]) to the operator $W$ we obtain that there exists a point $x_{0}^{\prime}$ of $\partial D$ such that

$$
\left\{\begin{array}{l}
u\left(x_{0}^{\prime}\right)=m, \\
u(x)<u\left(x_{0}^{\prime}\right) \quad \text { for all } x \in D .
\end{array}\right.
$$

Furthermore it follows from an application of the boundary point lemma (see [BC-P, Théorème VIII]) that

$$
\frac{\partial u}{\partial \mathbf{n}}\left(x_{0}^{\prime}\right)<0
$$

Hence we have

$$
\mu\left(x_{0}^{\prime}\right)=0, \quad \gamma\left(x_{0}^{\prime}\right)=0,
$$

since $L u\left(x_{0}^{\prime}\right) \geq 0$. This contradicts hypothesis $(\mathrm{H})$.

The proof of Theorem 1 is now complete.

\section{Proof of Theorem 2.}

The proof of Theorem 2 is based on the following version of the Hille-Yosida theorem in terms of the maximum principle (see [B-C-P, Théorème de Hille-Yosida-Ray]):

Theorem 2.1. Let $\mathcal{A}$ be a linear operator from the space $C_{0}(\bar{D} \backslash M)$ into itself, and assume that:

$(\alpha)$ The domain $D(\mathcal{A})$ is dense in the space $C_{0}(\bar{D} \backslash M)$.

$(\beta)$ For any $u \in D(\mathcal{A})$ such that $\sup u>0$, there exists a point $x \in \bar{D} \backslash M$ such that $u(x)=\sup u$ and $\mathcal{A} u(x) \leq 0$.

$(\gamma)$ For all $\alpha>0$, the range $R(\mathcal{A}-\alpha I)$ is dense in the space $C_{0}(\bar{D} \backslash M)$.

Then the operator $\mathcal{A}$ is closable in the space $C_{0}(\bar{D} \backslash M)$, and its minimal closed extension $\overline{\mathcal{A}}$ generates a Feller semigroup $\left\{T_{t}\right\}_{t \geq 0}$ on $\bar{D} \backslash M$.

Proof of Theorem 2. We have only to verify conditions $(\alpha),(\beta)$ and $(\gamma)$ in Theorem 2.1 for the operator $\mathcal{W}$.

$(\gamma)$ We obtain from Theorem 1 (and its proof) that the mapping

$$
(W-\alpha, L): C^{2+\theta}(\bar{D}) \longrightarrow C^{\theta}(\bar{D}) \oplus C_{L}^{1+\theta}(\partial D)
$$

is an algebraic and topological isomorphism for all $\alpha>0$. This verifies condition $(\gamma)$, since the range $R(\mathcal{W}-\alpha I)$ contains the space $C^{\theta}(\bar{D}) \cap C_{0}(\bar{D} \backslash M)$ which is dense in $C_{0}(\bar{D} \backslash M)$.

$(\beta)$ First let $x_{0}$ be a point of $D$ such that $u\left(x_{0}\right)=\sup u$. Then it follows from an application of $[\mathrm{B}-\mathrm{C}-\mathrm{P}$, Théorème $\mathrm{V}]$ that

$$
\mathcal{W} u\left(x_{0}\right)=W u\left(x_{0}\right) \leq 0
$$

Next let $x_{0}^{\prime}$ be a point of $\partial D \backslash M$ such that $u\left(x_{0}^{\prime}\right)=\sup u$. Assume to the contrary that

$$
\mathcal{W} u\left(x_{0}^{\prime}\right)=W u\left(x_{0}^{\prime}\right)>0
$$


We have only to consider the case where $u$ is not a constant. Then it follows from an application of the boundary point lemma that $(\partial u / \partial \mathbf{n})\left(x_{0}^{\prime}\right)<0$. Hence we have

$$
\mu\left(x_{0}^{\prime}\right)=0
$$

since $L u\left(x_{0}^{\prime}\right)=0$. This contradicts the hypothesis: $x_{0}^{\prime} \in \partial D \backslash M$, that is, $\mu\left(x_{0}^{\prime}\right)>0$.

$(\alpha)$ The density of the domain $D(\mathcal{W})$ can be proved just as in the proof of [T2, Theorem 8.20], by using [B-C-P, Proposition III.1.6].

The proof of Theorem 2 is complete.

\section{Proof of Theorem 3.}

The next theorem, which is a generalization of [T2, Theorem 6.1] to the integrodifferential operator case, proves Theorem 3:

Theorem 3.1. If hypothesis $(H)$ is satisfied, then, for every $0<\varepsilon<\pi / 2$, there exists a constant $r_{p}(\varepsilon)>0$ such that the resolvent set of $W_{p}$ contains the set $\Sigma_{p}(\varepsilon)=$ $\left\{\lambda=r^{2} e^{i \vartheta}: r \geq r_{p}(\varepsilon),-\pi+\varepsilon \leq \vartheta \leq \pi-\varepsilon\right\}$, and that the resolvent $\left(W_{p}-\lambda I\right)^{-1}$ satisfies estimate (0.3).

Proof. The proof is divided into three steps.

(i) We show that there exist constants $r_{p}(\varepsilon)$ and $c_{p}(\varepsilon)$ such that we have, for all $\lambda=r^{2} e^{i \vartheta}$ satisfying $r \geq r_{p}(\varepsilon)$ and $-\pi+\varepsilon \leq \vartheta \leq \pi+\varepsilon$,

$$
|u|_{2, p}+|\lambda|^{1 / 2}|u|_{1, p}+|\lambda|\|u\|_{p} \leq c_{p}(\varepsilon)\left\|\left(W_{p}-\lambda I\right) u\right\|_{p}
$$

Here

$$
\|u\|_{p}=\|u\|_{L^{p}(D)}, \quad|u|_{1, p}=\|\nabla u\|_{L^{p}(D)}, \quad|u|_{2, p}=\left\|\nabla^{2} u\right\|_{L^{p}(D)} .
$$

First we recall (see [T2, formula (6.2)]) that estimate (3.1) is proved for the differential operator $A$ :

$$
|u|_{2, p}+|\lambda|^{1 / 2}|u|_{1, p}+|\lambda|\|u\|_{p} \leq c_{p}^{\prime}(\varepsilon)\left\|\left(A_{p}-\lambda I\right) u\right\|_{p}
$$

Here the operator $A_{p}$ is a unbounded linear operator from $L^{p}(D)$ into itself defined by the following:

(a) The domain of definition $D\left(A_{p}\right)$ is the set

$$
D\left(A_{p}\right)=\left\{u \in H^{2, p}(D): L u=0\right\} .
$$

(b) $A_{p} u=A u, u \in D\left(A_{p}\right)$.

In order to replace the last term $\left\|\left(A_{p}-\lambda I\right) u\right\|_{p}$ by the term $\left\|\left(W_{p}-\lambda I\right) u\right\|_{p}$, we need the following $L^{p}$-estimate for the operator $S$ :

Lemma 3.2. For every $\eta>0$, there exists a constant $C_{\eta}>0$ such that we have, for all $u \in H^{2, p}(D)$,

$$
\|S u\|_{p} \leq \eta|u|_{2, p}+C_{\eta}\left(\|u\|_{p}+|u|_{1, p}\right)
$$

Proof. We decompose the term $S u$ into the following three terms:

$$
S u(x)
$$




$$
\begin{aligned}
= & \int_{0}^{1}(1-t) d t \int_{\{|z| \leq \varepsilon\}} z \cdot \nabla^{2} u(x+t z) z s(x, z) m(d z) \\
& +\int_{\{|z|>\varepsilon\}}(u(x+z)-u(x)) s(x, z) m(d z)-\int_{\{|z|>\varepsilon\}} z \cdot \nabla u(x) s(x, z) m(d z) \\
:= & S_{1} u(x)+S_{2} u(x)-S_{3} u(x) .
\end{aligned}
$$

First we estimate the $L^{p}$ norm of the term $S_{3} u$. By using estimate (1.2), we obtain that

$$
\left|\int_{\{|z|>\varepsilon\}} z \cdot \nabla u(x) s(x, z) m(d z)\right| \leq \delta(\varepsilon)|\nabla u(x)| \leq\left(\frac{C_{1}}{\varepsilon}+C_{2}\right)|\nabla u(x)| .
$$

Hence we have the $L^{p}$ estimate of the term $S_{3} u$ :

$$
\left\|S_{3} u\right\|_{p} \leq\left(\frac{C_{1}}{\varepsilon}+C_{2}\right)\|\nabla u\|_{p}
$$

Secondly we have

$$
\left\|\int_{\{|z|>\varepsilon\}} u(\cdot) s(\cdot, z) m(d z)\right\|_{p} \leq\left(\frac{C_{1}}{\varepsilon^{2}}+C_{2}\right)\|u\|_{p} .
$$

Furthermore, by using Hölder's inequality and Fubini's theorem we obtain from condition (0.1) that

$$
\begin{aligned}
& \int_{\mathbf{R}^{N}}\left|\int_{\{|z|>\varepsilon\}} u(x+z) s(x, z) m(d z)\right|^{p} d x \\
\leq & \int_{\mathbf{R}^{N}}\left(\int_{\{|z|>\varepsilon\}}|u(x+z)| s(x, z) m(d z)\right)^{p} d x \\
\leq & \int_{\mathbf{R}^{N}}\left(\int_{\{|z|>\varepsilon\}}|u(x+z)|^{p} s(x, z)^{p} m(d z)\right)\left(\int_{\{|z|>\varepsilon\}} m(d z)\right)^{p / q} d x \\
= & \tau(\varepsilon)^{p / q} \int_{\mathbf{R}^{N}} \int_{\{|z|>\varepsilon\}}|u(x+z)|^{p} s(x, z)^{p} m(d z) d x \\
= & \tau(\varepsilon)^{p / q} \int_{\{|z|>\varepsilon\}}\left(\int_{\mathbf{R}^{N}}|u(x+z)|^{p} s(x, z)^{p} d x\right) m(d z) \\
\leq & \tau(\varepsilon)^{p / q}\left(\int_{D}|u(y)|^{p} d y\right)\left(\int_{\{|z|>\varepsilon\}} m(d z)\right) \\
= & \tau(\varepsilon)^{p}\|u\|_{p}^{p} .
\end{aligned}
$$

By estimate (1.3), we have the $L^{p}$ estimate of the term $S_{2} u$ :

$$
\left\|S_{2} u\right\|_{p} \leq\left(\frac{C_{1}}{\varepsilon^{2}}+C_{2}\right)\|u\|_{p}
$$


Similarly, by using Hölder's inequality and Fubini's theorem we find that

$$
\begin{aligned}
& \int_{\mathbf{R}^{N}}\left|\int_{0}^{1}(1-t) d t \int_{\{|z| \leq \varepsilon\}} z \cdot \nabla^{2} u(x+t z) z s(x, z) m(d z)\right|^{p} d x \\
\leq & \int_{\mathbf{R}^{N}}\left(\int_{0}^{1} d t \int_{\{|z| \leq \varepsilon\}}|z|^{2}\left|\nabla^{2} u(x+t z)\right| s(x, z) m(d z)\right)^{p} d x \\
\leq & \int_{\mathbf{R}^{N}} \int_{0}^{1} d t\left(\int_{\{|z| \leq \varepsilon\}}|z|^{2}\left|\nabla^{2} u(x+t z)\right|^{p} s(x, z)^{p} m(d z)\right) \\
& \times\left(\int_{\{|z| \leq \varepsilon\}}|z|^{2} m(d z)\right)^{p / q} d x \\
= & \sigma(\varepsilon)^{p / q} \int_{\mathbf{R}^{N}} \int_{0}^{1} d t\left(\int_{\{|z| \leq \varepsilon\}}|z|^{2}\left|\nabla^{2} u(x+t z)\right|^{p} s(x, z)^{p} m(d z)\right) d x \\
= & \sigma(\varepsilon)^{p / q} \int_{0}^{1} d t \int_{\{|z| \leq \varepsilon\}}|z|^{2}\left(\int_{\mathbf{R}^{N}}\left|\nabla^{2} u(x+t z)\right|^{p} s(x, z)^{p} d x\right) m(d z) \\
\leq & \sigma(\varepsilon)^{p / q}\left(\int_{D}\left|\nabla^{2} u(y)\right|^{p} d y\right)\left(\int_{\{|z| \leq \varepsilon\}}|z|^{2} m(d z)\right) \\
\leq & \sigma(\varepsilon)^{p}\left(\int_{D}\left|\nabla^{2} u(y)\right|^{p} d y\right) \cdot
\end{aligned}
$$

Hence we have the $L^{p}$ estimate of the term $S_{1} u$ :

$$
\left\|S_{1} u\right\|_{p} \leq \sigma(\varepsilon)\left\|\nabla^{2} u\right\|_{p}
$$

Summing up, we have proved that

$$
\begin{aligned}
\|S u\|_{p} & \leq\left\|S_{1} u\right\|_{p}+\left\|S_{2} u\right\|_{p}+\left\|S_{3} u\right\|_{p} \\
& \leq \sigma(\varepsilon)|u|_{2, p}+\left(\frac{C_{1}}{\varepsilon}+C_{2}\right)|u|_{1, p}+\left(\frac{C_{1}}{\varepsilon^{2}}+C_{2}\right)\|u\|_{p} .
\end{aligned}
$$

In view of assertion (1.1), this proves estimate (3.3) if we choose $\varepsilon$ sufficiently small.

Since we have

$$
(A-\lambda) u=(W-\lambda) u-S u
$$

it follows from estimate (3.3) that

$$
\left\|\left(A_{p}-\lambda\right) u\right\|_{p} \leq\left\|\left(W_{p}-\lambda\right) u\right\|_{p}+\eta|u|_{2, p}+C_{\eta}\left(|u|_{1, p}+\|u\|_{p}\right) .
$$

Thus, carrying this estimate into estimate (3.2) we obtain that

$$
\begin{aligned}
& |u|_{2, p}+|\lambda|^{1 / 2}|u|_{1, p}+|\lambda|\|u\|_{p} \\
\leq & c_{p}^{\prime}(\varepsilon)\left\|\left(W_{p}-\lambda\right) u\right\|_{p}+\eta c_{p}^{\prime}(\varepsilon)|u|_{2, p}+C_{\eta} c_{p}^{\prime}(\varepsilon)\left(|u|_{1, p}+\|u\|_{p}\right) .
\end{aligned}
$$


Therefore, the desired estimate (3.1) follows from estimate (3.4) if we take the constant $\eta$ so small that

$$
\eta c_{p}^{\prime}(\varepsilon)<1
$$

and the parameter $\lambda$ so large that

$$
|\lambda|^{1 / 2}>C_{\eta} c_{p}^{\prime}(\varepsilon)
$$

(ii) By estimate (3.1), we find that the operator $W_{p}-\lambda I$ is injective and its range $R\left(W_{p}-\lambda I\right)$ is closed in $L^{p}(D)$, for all $\lambda \in \Sigma_{p}(\varepsilon)$.

We show that the operator $W_{p}-\lambda I$ is surjective for all $\lambda \in \Sigma_{p}(\varepsilon)$ :

$$
R\left(W_{p}-\lambda I\right)=L^{p}(D), \quad \lambda \in \Sigma_{p}(\varepsilon) .
$$

To do so, it suffices to show that the operator $W_{p}-\lambda I$ is a Fredholm operator with

$$
\text { ind }\left(W_{p}-\lambda I\right)=0, \quad \lambda \in \Sigma_{p}(\varepsilon),
$$

since $W_{p}-\lambda I$ is injective for all $\lambda \in \Sigma_{p}(\varepsilon)$.

In order to prove assertion (3.5), we need the following:

Lemma 3.3. The operator $S$ is $A_{p}$-completely continuous, that is, the operator $S: D\left(A_{p}\right) \rightarrow L^{p}(D)$ is completely continuous where the domain $D\left(A_{p}\right)$ is endowed with the graph norm of $A_{p}$.

Proof. Let $\left\{u_{j}\right\}$ be an arbitrary bounded sequence in the domain $D\left(A_{p}\right)$; hence there exists a constant $K>0$ such that

$$
\left\|u_{j}\right\|_{p} \leq K, \quad\left\|A_{p} u_{j}\right\|_{p} \leq K .
$$

Then we have, by [T2, estimate $(0.1)]$,

$$
\left\|u_{j}\right\|_{2, p} \leq C\left(\left\|A_{p} u_{j}\right\|_{p}+\left\|u_{j}\right\|_{p}\right) \leq 2 C K .
$$

Therefore, by Rellich's theorem one may assume that the sequence $\left\{u_{j}\right\}$ itself is a Cauchy sequence in the space $H^{1, p}(D)$. Then, applying estimate (3.3) to the sequence $\left\{u_{j}-u_{k}\right\}$ and using estimate (3.6), we obtain that

$$
\begin{aligned}
\left\|S u_{j}-S u_{k}\right\|_{p} & \leq \eta\left|u_{j}-u_{k}\right|_{2, p}+C_{\eta}\left(\left\|u_{j}-u_{k}\right\|_{p}+\left|u_{j}-u_{k}\right|_{1, p}\right) \\
& \leq 4 \eta C K+C_{\eta}\left\|u_{j}-u_{k}\right\|_{1, p} .
\end{aligned}
$$

Hence we have

$$
\limsup _{j, k \rightarrow \infty}\left\|S u_{j}-S u_{k}\right\|_{p} \leq 4 \eta C K .
$$

This proves that the sequence $\left\{S u_{j}\right\}$ is a Cauchy sequence in the space $L^{p}(D)$, since $\eta$ is arbitrary.

In view of Lemma 3.3, assertion (3.5) follows from an application of [G-K, Theorem 2.6]. Indeed we have, by [T2, Theorem 6.1],

$$
\text { ind }\left(W_{p}-\lambda I\right)=\operatorname{ind}\left(A_{p}-\lambda I+S\right)=\operatorname{ind}\left(A_{p}-\lambda I\right)=0 \text {. }
$$

(iii) Summing up, we have proved that the operator $W_{p}-\lambda I$ is bijective for all $\lambda \in \Sigma_{p}(\varepsilon)$ and its inverse $\left(W_{p}-\lambda I\right)^{-1}$ satisfies estimate $(0.3)$.

The proof of Theorem 3.1 is now complete. 


\section{Proof of Theorem 4.}

The proof is carried out in a chain of auxiliary lemmas.

(I) We begin with a version of estimate (3.1):

Lemma 4.1. Let $N<p<\infty$. If hypothesis $(H)$ is satisfied, then, for every $\varepsilon>0$, there exists a constant $r_{p}(\varepsilon)>0$ such that if $\lambda=r^{2} e^{i \vartheta}$ with $r \geq r_{p}(\varepsilon)$ and $-\pi+\varepsilon \leq \vartheta \leq \pi-\varepsilon$, we have, for all $u \in D\left(W_{p}\right)$,

$$
|\lambda|^{1 / 2}\|u\|_{C^{1}(\bar{D})}+|\lambda|\|u\|_{C(\bar{D})} \leq C_{p}(\varepsilon)|\lambda|^{N / 2 p}\|(W-\lambda) u\|_{p}
$$

with a constant $C_{p}(\varepsilon)>0$.

Proof. First it follows from an application of the Gagliardo-Nirenberg inequality (see [F, Part I, Theorem 10.1] that

$$
\|u\|_{C(\bar{D})} \leq C|u|_{1, p}^{N / p}\|u\|_{p}^{1-N / p}, \quad u \in H^{1, p}(D)
$$

Here and in the following the letter $C$ denotes a generic positive constant depending on $p$ and $\varepsilon$, but independent of $u$ and $\lambda$.

Combining inequality (4.2) with inequality (3.1), we obtain that

$$
\begin{aligned}
\|u\|_{C(\bar{D})} & \leq C\left(|\lambda|^{-1 / 2}\|(W-\lambda) u\|_{p}\right)^{N / p}\left(|\lambda|^{-1}\|(W-\lambda) u\|_{p}\right)^{1-N / p} \\
& =C|\lambda|^{-1+N / 2 p}\|(W-\lambda) u\|_{p}
\end{aligned}
$$

so that

$$
|\lambda|\|u\|_{C(\bar{D})} \leq C|\lambda|^{N / 2 p}\|(W-\lambda) u\|_{p}, \quad u \in D\left(W_{p}\right)
$$

Similarly, applying inequality (4.2) to the functions $D_{i} u \in H^{1, p}(D)(1 \leq i \leq n)$ we obtain that

$$
\begin{aligned}
\|\nabla u\|_{C(\bar{D})} & \leq C|\nabla u|_{1, p}^{N / p}\|\nabla u\|_{p}^{1-N / p} \\
& \leq C|u|_{2, p}^{N / p}|u|_{1, p}^{1-N / p} \\
& \leq C\left(\|(W-\lambda) u\|_{p}\right)^{N / p}\left(|\lambda|^{-1 / 2}\|(W-\lambda) u\|_{p}\right)^{1-N / p} \\
& =C|\lambda|^{-1 / 2+N / 2 p}\|(W-\lambda) u\|_{p} .
\end{aligned}
$$

This proves that

$$
|\lambda|^{1 / 2}\|u\|_{C^{1}(\bar{D})} \leq C|\lambda|^{N / 2 p}\|(W-\lambda) u\|_{p}, \quad u \in D\left(W_{p}\right)
$$

Therefore, the desired inequality (4.1) follows from inequalities (4.3) and (4.4).

(II) The next lemma proves estimate (0.4): 
Lemma 4.2. Let $N<p<\infty$. If hypothesis $(H)$ is satisfied, then, for every $\varepsilon>0$, there exists a constant $r(\varepsilon)>0$ such that if $\lambda=r^{2} e^{i \vartheta}$ with $r \geq r(\varepsilon)$ and $-\pi+\varepsilon \leq \vartheta \leq \pi-\varepsilon$, we have, for all $u \in D(\mathfrak{W})$,

$$
|\lambda|^{1 / 2}\|u\|_{C^{1}(\bar{D})}+|\lambda|\|u\|_{C(\bar{D})} \leq c(\varepsilon)\|(\mathfrak{W}-\lambda I) u\|_{C(\bar{D})},
$$

with a constant $c(\varepsilon)>0$.

Proof. (1) First we show that the domain

$$
D(\mathfrak{W})=\left\{u \in C_{0}(\bar{D} \backslash M) \cap H^{2, p}(D): W u \in C_{0}(\bar{D} \backslash M), L u=0\right\}
$$

is independent of $N<p<\infty$.

We let

$$
\mathcal{D}_{p}=\left\{u \in H^{2, p}(D) \cap C_{0}(\bar{D} \backslash M): W u \in C_{0}(\bar{D} \backslash M), L u=0\right\} .
$$

Since we have $L^{p_{1}}(D) \subset L^{p_{2}}(D)$ for $p_{1}>p_{2}$, it follows that

$$
\mathcal{D}_{p_{1}} \subset \mathcal{D}_{p_{2}} \text { if } p_{1}>p_{2} .
$$

Conversely, let $v$ be an arbitrary element of $\mathcal{D}_{p_{2}}$ :

$$
v \in H^{2, p_{2}}(D) \cap C_{0}(\bar{D} \backslash M), \quad W v \in C_{0}(\bar{D} \backslash M), \quad L v=0 .
$$

Then, since we have $v, W v \in C_{0}(\bar{D} \backslash M) \subset L^{p_{1}}(D)$, it follows from an application of Theorem 3.1 with $p=p_{1}$ that there exists a unique function $u \in H^{2, p_{1}}(D)$ such that

$$
\begin{cases}(W-\lambda) u=(W-\lambda) v & \text { in } D, \\ L u=0 & \text { on } \partial D,\end{cases}
$$

if we choose $\lambda$ sufficiently large. Hence we have $u-v \in H^{2, p_{2}}(D)$ and

$$
\begin{cases}(W-\lambda)(u-v)=0 & \text { in } D \\ L(u-v)=0 & \text { on } \partial D .\end{cases}
$$

Therefore, by applying again Theorem 3.1 with $p=p_{2}$ we obtain that $u-v=0$, so that $v=u \in H^{2, p_{1}}(D)$. This proves that $v \in \mathcal{D}_{p_{1}}$.

(2) We shall make use of a $\lambda$-dependent localization argument in order to adjust the term $\|(W-\lambda) u\|_{p}$ in inequality (4.1) to obtain inequality (4.5), just as in [T2].

(2-a) If $x_{0}^{\prime}$ is a point of $\partial D$ and if $\chi$ is a $C^{\infty}$ coordinate transformation such that $\chi$ maps $B\left(x_{0}^{\prime}, \eta_{0}\right) \cap D$ into $B(0, \delta) \cap \mathbf{R}_{+}^{N}$ and flattens a part of the boundary $\partial D$ into the plane $x_{N}=0$, then we let

$$
\begin{aligned}
& G_{0}=B\left(x_{0}^{\prime}, \eta_{0}\right) \cap D, \\
& G^{\prime}=B\left(x_{0}^{\prime}, \eta\right) \cap D, 0<\eta<\eta_{0}, \\
& G^{\prime \prime}=B\left(x_{0}^{\prime}, \eta / 2\right) \cap D, 0<\eta<\eta_{0} .
\end{aligned}
$$

Here and in the following $B(x, \eta)$ denotes the ball of radius $\eta$ about $x$. 
Similarly, if $x_{0}$ is a point of $D$ and if $\chi$ is a $C^{\infty}$ coordinate transformation such that $\chi$ maps $B\left(x_{0}, \eta_{0}\right)$ into $B(0, \delta)$, then we let

$$
\begin{aligned}
& G_{0}=B\left(x_{0}, \eta_{0}\right), \\
& G^{\prime}=B\left(x_{0}, \eta\right), 0<\eta<\eta_{0}, \\
& G^{\prime \prime}=B\left(x_{0}, \eta / 2\right), 0<\eta<\eta_{0} .
\end{aligned}
$$

(2-b) We take a function $\Phi \in C_{0}^{\infty}(\mathbf{R})$ such that $\Phi$ equals 1 near the origin, and define

$$
\varphi(x)=\Phi\left(\left|x^{\prime}\right|^{2}\right) \Phi\left(x_{N}\right), \quad x=\left(x^{\prime}, x_{N}\right) .
$$

Here one may assume that the function $\varphi$ is chosen so that

$$
\left\{\begin{array}{l}
\operatorname{supp} \varphi \subset B(0,1), \\
\varphi(x)=1 \text { on } B(0,1 / 2) .
\end{array}\right.
$$

We introduce a localizing function

$$
\varphi_{0}(x, \eta):=\varphi\left(\frac{x-x_{0}}{\eta}\right)=\Phi\left(\frac{\left|x^{\prime}-x_{0}^{\prime}\right|^{2}}{\eta^{2}}\right) \Phi\left(\frac{x_{N}-t}{\eta}\right), \quad x_{0}=\left(x_{0}^{\prime}, t\right) .
$$

We remark that

$$
\left\{\begin{array}{l}
\operatorname{supp} \varphi_{0} \subset B\left(x_{0}, \eta\right), \\
\varphi_{0}(x, \eta)=1 \text { on } B\left(x_{0}, \eta / 2\right) .
\end{array}\right.
$$

Then it is easy to verify the following (cf. [T2, Claim 7.5]):

Claim 4.3. If $u \in D(\mathfrak{W})$, then we have $\varphi_{0} u \in \mathcal{D}\left(W_{p}\right)$.

(3) Now let $u$ be an arbitrary element of $D(\mathfrak{W})$. Then, by Claim 4.3 we can apply inequality (4.1) to the function $\varphi_{0} u$ to obtain that

$$
\begin{aligned}
|\lambda|^{1 / 2}\|u\|_{C^{1}\left(\overline{G^{\prime \prime}}\right)}+|\lambda|\|u\|_{C\left(\overline{G^{\prime \prime}}\right)} & \leq|\lambda|^{1 / 2}\left\|\varphi_{0} u\right\|_{C^{1}\left(\overline{G^{\prime}}\right)}+|\lambda|\left\|\varphi_{0} u\right\|_{C\left(\overline{G^{\prime}}\right)} \\
& =|\lambda|^{1 / 2}\left\|\varphi_{0} u\right\|_{C^{1}(\bar{D})}+|\lambda|\left\|\varphi_{0} u\right\|_{C(\bar{D})} \\
& \leq C|\lambda|^{N / 2 p}\left\|(W-\lambda)\left(\varphi_{0} u\right)\right\|_{L^{p}(D)} .
\end{aligned}
$$

(3-a) We estimate the last term $\left\|(W-\lambda)\left(\varphi_{0} u\right)\right\|_{L^{p}(D)}$ in terms of the supremum norm of $C(\bar{D})$.

First we write the term $(W-\lambda)\left(\varphi_{0} u\right)$ in the following form:

$$
(W-\lambda)\left(\varphi_{0} u\right)=\varphi_{0}((W-\lambda) u)+\left[A, \varphi_{0}\right] u+\left[S, \varphi_{0}\right] u,
$$

where $\left[A, \varphi_{0}\right]$ and $\left[S, \varphi_{0}\right]$ are the commutators of $A$ and $\varphi_{0}$ and of $S$ and $\varphi_{0}$, respectively:

$$
\begin{aligned}
& {\left[A, \varphi_{0}\right] u=A\left(\varphi_{0} u\right)-\varphi_{0} A u} \\
& {\left[S, \varphi_{0}\right] u=S\left(\varphi_{0} u\right)-\varphi_{0} S u .}
\end{aligned}
$$

Now we need the following elementary inequality: 
Claim 4.4. We have, for all $v \in C^{j}\left(\overline{G^{\prime}}\right)(j=0,1,2)$,

$$
\|v\|_{H^{j, p}\left(G^{\prime}\right)} \leq\left|G^{\prime}\right|^{1 / p}\|v\|_{C^{j}\left(\overline{G^{\prime}}\right)},
$$

where $\left|G^{\prime}\right|$ is the measure of $G^{\prime}$.

Since we have, for some constant $c>0$,

$$
\left|G^{\prime}\right| \leq\left|B\left(x_{0}, \eta\right)\right| \leq c \eta^{N}
$$

it follows from an application of Claim 4.4 that

$$
\begin{aligned}
\left\|\varphi_{0}(W-\lambda) u\right\|_{L^{p}(D)} & =\left\|\varphi_{0}(W-\lambda) u\right\|_{L^{p}\left(G^{\prime}\right)} \\
& \leq c^{1 / p} \eta^{N / p}\|(W-\lambda) u\|_{C\left(\overline{G^{\prime}}\right)} \\
& \leq c^{1 / p} \eta^{N / p}\|(W-\lambda) u\|_{C(\bar{D})}
\end{aligned}
$$

On the other hand we can estimate the commutators $\left[A, \varphi_{0}\right] u$ and $\left[S, \varphi_{0}\right] u$ as follows:

Claim 4.5. We have, as $\eta \downarrow 0$,

$$
\begin{aligned}
& \left\|\left[A, \varphi_{0}\right] u\right\|_{L^{p}(D)} \leq C\left(\eta^{-1+N / p}\|u\|_{C^{1}(\bar{D})}+\eta^{-2+N / p}\|u\|_{C(\bar{D})}\right) \\
& \left\|\left[S, \varphi_{0}\right] u\right\|_{L^{p}(D)} \leq C\left(\eta^{-1+N / p}\|u\|_{C^{1}(\bar{D})}+\eta^{-2+N / p}\|u\|_{C(\bar{D})}\right)
\end{aligned}
$$

Proof. Estimate (4.8) is proved in [T2, inequality (7.9)].

In order to prove estimate (4.9), we remark that

$$
\begin{aligned}
& S\left(\varphi_{0} u\right)(x) \\
= & \int_{\mathbf{R}^{N} \backslash\{0\}}\left(\varphi_{0}(x+z) u(x+z)-\varphi_{0}(x) u(x)-z \cdot \nabla\left(\varphi_{0} u\right)(x)\right) s(x, z) m(d z) \\
= & \varphi_{0}(x) \int_{\mathbf{R}^{N} \backslash\{0\}}(u(x+z)-u(x)-z \cdot \nabla u(x)) s(x, z) m(d z) \\
& \left.+\int_{\mathbf{R}^{N} \backslash\{0\}}(u(x+z)-u(x)) z s(x, z) m(d z)\right) \cdot \nabla \varphi_{0}(x) \\
& +\int_{\mathbf{R}^{N} \backslash\{0\}}\left(\varphi_{0}(x+z)-\varphi_{0}(x)-z \cdot \nabla \varphi_{0}(x)\right) u(x+z) s(x, z) m(d z) \\
= & \varphi_{0}(x) S u(x)+\left(\int_{\mathbf{R}^{N} \backslash\{0\}}(u(x+z)-u(x)) z s(x, z) m(d z)\right) \cdot \nabla \varphi_{0}(x) \\
& +\int_{\mathbf{R}^{N} \backslash\{0\}}\left(\varphi_{0}(x+z)-\varphi_{0}(x)-z \cdot \nabla \varphi_{0}(x)\right) u(x+z) s(x, z) m(d z) .
\end{aligned}
$$

Hence we can write the commutator $\left[S, \varphi_{0}\right] u$ in the following form:

$$
\left[S, \varphi_{0}\right] u(x)
$$




$$
\begin{aligned}
= & \left(\int_{\mathbf{R}^{N} \backslash\{0\}}(u(x+z)-u(x)) z s(x, z) m(d z)\right) \cdot \nabla \varphi_{0}(x) \\
& +\int_{\mathbf{R}^{N} \backslash\{0\}}\left(\varphi_{0}(x+z)-\varphi_{0}(x)-z \cdot \nabla \varphi_{0}(x)\right) u(x+z) s(x, z) m(d z) \\
:= & S_{0}^{(1)} u(x)+S_{0}^{(2)} u(x) .
\end{aligned}
$$

First, just as in Lemma 1.6 we can estimate the term $S_{0}^{(1)} u$ as follows:

$$
\begin{aligned}
\left\|S_{0}^{(1)} u\right\|_{L^{p}(D)} & =\left\|S_{0}^{(1)} u\right\|_{L^{p}\left(G^{\prime}\right)} \\
& \leq 2\left(\sigma(\eta)\|u\|_{C^{1}(\bar{D})}+\delta(\eta)\|u\|_{C(\bar{D})}\right)\left\|\nabla \varphi_{0}\right\|_{L^{p}\left(G^{\prime}\right)} \\
& \leq 2\left(\sigma(\eta)\|u\|_{C^{1}(\bar{D})}+\left(\frac{C_{1}}{\eta}+C_{2}\right)\|u\|_{C(\bar{D})}\right)\left\|\nabla \varphi_{0}\right\|_{L^{p}\left(G^{\prime}\right)} .
\end{aligned}
$$

However it follows from an application of Claim 4.4 that

$$
\begin{aligned}
& \left\|\nabla \varphi_{0}\right\|_{L^{p}\left(G^{\prime}\right)} \leq C \eta^{N / p}\left\|\nabla \varphi_{0}\right\|_{C\left(\overline{G^{\prime}}\right)} \leq C^{\prime} \eta^{-1+N / p} \\
& \left\|\nabla^{2} \varphi_{0}\right\|_{L^{p}\left(G^{\prime}\right)} \leq C \eta^{N / p}\left\|\nabla^{2} \varphi_{0}\right\|_{C\left(\overline{G^{\prime}}\right)} \leq C^{\prime} \eta^{-2+N / p}
\end{aligned}
$$

since we have, as $\eta \downarrow 0$,

$$
\left|\nabla \varphi_{0}\right|=O\left(\eta^{-1}\right), \quad\left|\nabla^{2} \varphi_{0}\right|=O\left(\eta^{-2}\right)
$$

Therefore we obtain that

$$
\left\|S_{0}^{(1)} u\right\|_{L^{p}(D)} \leq C\left(\eta^{-1+N / p}\|u\|_{C^{1}(\bar{D})}+\eta^{-2+N / p}\|u\|_{C(\bar{D})}\right) .
$$

Similarly, arguing as in the proof of Lemma 3.2 we can estimate the term $S_{0}^{(2)} u$ as follows:

$$
\begin{aligned}
\left\|S_{0}^{(2)} u\right\|_{L^{p}(D)} & \leq C\|u\|_{C(\bar{D})}\left\|\nabla^{2} \varphi_{0}\right\|_{L^{p}\left(G^{\prime}\right)} \\
& \leq C\|u\|_{C(\bar{D})} \eta^{N / p}\left\|\nabla^{2} \varphi_{0}\right\|_{C\left(\overline{G^{\prime}}\right)} \\
& \leq C \eta^{-2+N / p}\|u\|_{C(\bar{D})} .
\end{aligned}
$$

Thus, the desired estimate (4.9) follows by combining estimates (4.10) and (4.11).

Therefore, combining estimates (4.6), (4.7), (4.8) and (4.9) we obtain that

$$
\begin{aligned}
& |\lambda|^{1 / 2}\|u\|_{C^{1}\left(\overline{G^{\prime \prime}}\right)}+|\lambda|\|u\|_{C\left(\overline{G^{\prime \prime}}\right)} \\
\leq & C|\lambda|^{N / 2 p}\left\|(W-\lambda)\left(\varphi_{0} u\right)\right\|_{L^{p}(D)} \\
= & C|\lambda|^{N / 2 p}\left\|\varphi_{0}((W-\lambda) u)+\left[A, \varphi_{0}\right] u+\left[S, \varphi_{0}\right] u\right\|_{L^{p}(D)} \\
\leq & C|\lambda|^{N / 2 p}\left(\eta^{N / p}\|(W-\lambda) u\|_{C\left(\overline{G^{\prime}}\right)}+\eta^{-1+N / p}\|u\|_{C^{1}\left(\overline{G^{\prime}}\right)}+\eta^{-2+N / p}\|u\|_{C\left(\overline{G^{\prime}}\right)}\right) \\
\leq & C|\lambda|^{N / 2 p}\left(\eta^{N / p}\|(W-\lambda) u\|_{C(\bar{D})}+\eta^{-1+N / p}\|u\|_{C^{1}(\bar{D})}+\eta^{-2+N / p}\|u\|_{C(\bar{D})}\right) .
\end{aligned}
$$


(3-b) We remark that the closure $\bar{D}=D \cup \partial D$ can be covered by a finite number of sets of the forms:

$$
\begin{cases}B\left(x_{0}, \eta / 2\right), & x_{0} \in D, \\ B\left(x_{0}^{\prime}, \eta / 2\right) \cap \bar{D}, & x_{0}^{\prime} \in \partial D .\end{cases}
$$

Therefore, taking the supremum of inequality (4.12) over $x \in \bar{D}$ we find that

$$
\begin{aligned}
& |\lambda|^{1 / 2}\|u\|_{C^{1}(\bar{D})}+|\lambda|\|u\|_{C(\bar{D})} \\
\leq & C|\lambda|^{N / 2 p} \eta^{N / p}\left(\|(W-\lambda) u\|_{C(\bar{D})}+\eta^{-1}\|u\|_{C^{1}(\bar{D})}+\eta^{-2}\|u\|_{C(\bar{D})}\right) .
\end{aligned}
$$

(4) We now choose the localization parameter $\eta$. We let

$$
\eta=\frac{\eta_{0}}{|\lambda|^{1 / 2}} K
$$

where $K$ is a positive constant (to be chosen later) satisfying

$$
0<\eta=\frac{\eta_{0}}{|\lambda|^{1 / 2}} K<\eta_{0},
$$

that is,

$$
0<K<|\lambda|^{1 / 2} .
$$

Then we obtain from inequality (4.13) that

$$
\begin{aligned}
& |\lambda|^{1 / 2}\|u\|_{C^{1}(\bar{D})}+|\lambda|\|u\|_{C(\bar{D})} \\
\leq & C \eta_{0}^{N / p} K^{N / p}\|(W-\lambda) u\|_{C(\bar{D})}+\left(C \eta_{0}^{N / p-1} K^{-1+N / p}\right)|\lambda|^{1 / 2}\|u\|_{C^{1}(\bar{D})} \\
& +\left(C \eta_{0}^{N / p-2} K^{-2+N / p}\right)|\lambda|\|u\|_{C(\bar{D})} .
\end{aligned}
$$

However, since the exponents $-1+N / p$ and $-2+N / p$ are negative, we can choose the constant $K$ so large that

$$
C \eta_{0}^{N / p-1} K^{-1+N / p}<1,
$$

and

$$
C \eta_{0}^{N / p-2} K^{-2+N / p}<1 .
$$

Then, the desired inequality (4.5) follows from inequality (4.14).

The proof of Lemma 4.2 is complete.

(III) The next lemma, together with Lemma 4.2, proves that the resolvent set of $\mathfrak{W}$ contains the set $\Sigma(\varepsilon)=\left\{\lambda=r^{2} e^{i \vartheta}: r \geq r(\varepsilon), \quad-\pi+\varepsilon \leq \vartheta \leq \pi-\varepsilon\right\}$ : 
Lemma 4.6. If $\lambda \in \Sigma(\varepsilon)$, then, for any $f \in C_{0}(\bar{D} \backslash M)$, there exists a unique function $u \in D(\mathfrak{W})$ such that $(\mathfrak{W}-\lambda I) u=f$.

Proof. Since we have, for all $1<p<\infty$,

$$
f \in C_{0}(\bar{D} \backslash M) \subset L^{p}(D)
$$

it follows from an application of Theorem 3 that if $\lambda \in \Sigma_{p}(\varepsilon)$, there exists a unique function $u \in H^{2, p}(D)$ such that

$$
(W-\lambda) u=f \quad \text { in } D
$$

and

$$
L u=\mu \frac{\partial u}{\partial \mathbf{n}}+\gamma u=0 \quad \text { on } \partial D .
$$

However, by Sobolev's imbedding theorem it follows that

$$
u \in H^{2, p}(D) \subset C^{2-N / p}(\bar{D}) \subset C^{1}(\bar{D}) \quad \text { if } \quad N<p<\infty .
$$

Hence we have, by formula (4.16) and condition $(H)$,

$$
u=0 \quad \text { on } \quad M=\left\{x^{\prime} \in \partial D: \mu\left(x^{\prime}\right)=0\right\},
$$

so that

$$
u \in C_{0}(\bar{D} \backslash M) .
$$

Further, in view of equation (4.15) we find that

$$
W u=f+\lambda u \in C_{0}(\bar{D} \backslash M) .
$$

Summing up, we have proved that

$$
\left\{\begin{array}{l}
u \in D(\mathfrak{W}), \\
(\mathfrak{W}-\lambda I) u=f .
\end{array}\right.
$$

Now the proof of Theorem 4 is complete.

\section{References}

[B-L] Bergh, J., Löfström, J., Interpolation spaces, an introduction, Springer-Verlag, Berlin, 1976.

[B-C-P] Bony, J.-M., Courrège, P., Priouret, P., Semi-groupes de Feller sur une variété à bord compacte et problèmes aux limites intégro-différentiels du second ordre donnant lieu au principe du maximum, Ann. Inst. Fourier (Grenoble) 18 (1968), 369-521.

[B] Bourdaud, G., $L^{p}$-estimates for certain non-regular pseudo-differential operators, Comm. in Partial Differential Equations 7 (1982), 1023-1033.

[F] Friedman, A., Partial differential equations, Holt, Rinehart and Winston, New York, 1969.

[G-M] Garroni, M. G., Menaldi, J. L., Green functions for second order integro-differential problems, Pitman Research Notes in Mathematics Series No. 275, Longman Scientific \& Technical, Harlow, 1992. 
[G-T] Gilbarg, D., Trudinger, N. S., Elliptic partial differential equations of second order, Springer-Verlag, New York Berlin Heidelberg Tokyo, 1983.

[G-K] Gohberg, I. C., Kreĭn, M. G., The basic propositions on defect numbers, root numbers and indices of linear operators, Uspehi Mat. Nauk. 12 (1957), 43-118 (in Russian); English translation Amer. Math. Soc. Transl. 13 (1960), 185-264.

[H] Hörmander, L., The analysis of linear partial differential operators III, Springer-Verlag, Berlin Heidelberg New York Tokyo, 1985.

[K] Komatsu, T., Markov processes associated with certain integro-differential operators, Osaka J. Math. 10 (1973), 271-303.

[S] Stroock, D. W., Diffusion processes associated with Lévy generators, Z. Wahrscheinlichkeitstheorie verw. Gebiete 32 (1975), 209-244.

[T1] Taira, K., Diffusion processes and partial differential equations, Academic Press, San Diego New York London Tokyo, 1988.

[T2] Taira, K., Boundary value problems and Markov processes, Lecture Notes in Math. No. 1499, Springer-Verlag, Berlin Heidelberg New York Tokyo, 1991. 